\title{
LIPSCHITZ REGULARITY FOR INNER-VARIATIONAL EQUATIONS
}

\author{
TADEUSZ IWANIEC, LEONID V. KOVALEV, AND JANI ONNINEN
}

\begin{abstract}
We obtain Lipschitz regularity results for a fairly general class of nonlinear first-order PDEs. These equations arise from the inner variation of certain energy integrals. Even in the simplest model case of the Dirichlet energy the inner-stationary solutions need not be differentiable everywhere; the Lipschitz continuity is the best possible. But the proofs, even in the Dirichlet case, turn out to relay on topological arguments. The appeal to the inner-stationary solutions in this context is motivated by the classical problems of existence and regularity of the energy-minimal deformations in the theory of harmonic mappings and certain mathematical models of nonlinear elasticity; specifically, neoHookian type problems.
\end{abstract}

\section{INTRODUCTION}

We establish Lipschitz regularity of solutions of nonlinear first-order PDEs that arise from inner variation of numerous energy integrals. This includes the model case of the Dirichlet energy for mappings $h: \Omega \rightarrow \Omega^{*}$ between two designated domains in $\mathbb{C}$. Roughly speaking, the inner variation of $h$ amounts to composing $h$ with a diffeomorphism of $\Omega$ onto itself. This type of variation is often used when the standard first variation is not allowed. For instance, when dealing with mappings with nonnegative Jacobian the inner variation is necessary to preserve the sign of the Jacobian. Let us begin with the Dirichlet integral,

$$
\mathscr{E}_{\Omega}[h]=\iint_{\Omega}|D h|^{2}=2 \iint_{\Omega}\left(\left|h_{z}\right|^{2}+\left|h_{\bar{z}}\right|^{2}\right) \mathrm{d} x_{1} \mathrm{~d} x_{2}, \quad z=x_{1}+i x_{2}
$$

Hereafter $h_{z}=\frac{\partial h}{\partial z}$ and $h_{\bar{z}}=\frac{\partial h}{\partial \bar{z}}$ are complex partial derivatives of $h$. The first variation of $\mathscr{E}$ results in the Euler-Lagrange equation,

$$
\Delta h=4 h_{z \bar{z}}=0
$$

2010 Mathematics Subject Classification. Primary 49N60; Secondary 35B65, 73C50.

Key words and phrases. Inner variation, Lipschitz regularity, extremal problems, quasiregular mappings, topological degree.

Iwaniec was supported by the NSF grant DMS-0800416 and the Academy of Finland project 1128331. Kovalev was supported by the NSF grant DMS-0968756. Onninen was supported by the NSF grant DMS-1001620. 
In contrast, the inner variation leads to a nonlinear equation

$$
\frac{\partial}{\partial \bar{z}}\left(h_{z} \overline{h_{\bar{z}}}\right)=0, \quad \text { equivalently, } \quad h_{z} \overline{h_{\bar{z}}}=\phi, \quad(\phi \text { is analytic })
$$

for mapping in the Sobolev space $\mathscr{W}_{\text {loc }}^{1,2}(\Omega)$. This equation will henceforth be referred to as the Hopf-Laplace equation. There are important nonharmonic solutions of (1.3). Such solutions arise typically as weak limits of the energyminimizing sequences of diffeomorphisms $h: \Omega \stackrel{\text { onto }}{\longrightarrow} \Omega^{*}$. Passing to the limit we often loose harmonicity; at the points where the limit map fails to be injective [16], and only at those points [17].

The unavailability of the Euler-Lagrange equation is a major source of difficulties in the theory of nonlinear elasticity [7, 8, 29]. This drives one to investigate the regularity of the energy-minimal mappings on the basis of the inner-variational equation alone; also known as energy-momentum or equilibrium equations, etc $[11,27,30]$. Several results in this direction were obtained in $[9,12,25,32]$. Nevertheless this theory is still in its infancy.

The most general setting we are dealing with can be described as follows. Let $\mathcal{H}=\mathcal{H}(z, \xi)$ be a continuous function in $\Omega \times \widehat{\mathbb{C}}_{R}$, where $\widehat{\mathbb{C}}_{R}=\{\xi: R<$ $|\xi| \leqslant \infty\}, 0 \leqslant R<\infty$. We impose two structural conditions on $\mathcal{H}$. The first one is the Lipschitz condition with respect to the reciprocal of the $\xi$ variable,

$$
\left|\mathcal{H}\left(z, \xi_{1}\right)-\mathcal{H}\left(z, \xi_{2}\right)\right| \leqslant L \cdot\left|\frac{1}{\xi_{1}}-\frac{1}{\xi_{2}}\right|, \text { for } z \in \Omega \text { and } \xi_{1}, \xi_{2} \in \widehat{\mathbb{C}}_{R} .
$$

with a constant $0 \leqslant L<\infty$. Regarding regularity with respect to $z \in \Omega$, we shall require that the function $z \mapsto \mathcal{H}(z, \xi)$ be Hölder continuous. Precisely, the second structural condition reads as:

$$
\sup _{z \in \Omega}|\mathcal{H}(z, \xi)|+\sup _{z_{1} \neq z_{2}} \frac{\left|\mathcal{H}\left(z_{1}, \xi\right)-\mathcal{H}\left(z_{2}, \xi\right)\right|}{\left|z_{1}-z_{2}\right|^{\alpha}} \leqslant M, \text { for }|\xi|>R
$$

Definition 1.1. A function $h \in \mathscr{W}_{\text {loc }}^{1,2}(\Omega)$ is said to be a solution of the equation

$$
h_{\bar{z}}=\mathcal{H}\left(z, h_{z}\right)
$$

if (1.6) holds for almost every point $z \in \Omega$, whenever $\left|h_{z}(z)\right|>R$.

Note we impose no condition at the points where $\left|h_{z}(z)\right| \leqslant R$; this yields boundedness of the gradient of $h,\left|h_{\bar{z}}\right| \leqslant\left|h_{z}\right| \leqslant R$.

A simplified version of our main result reads as follows

Theorem 1.2. Let the equation (1.6) comply with the conditions (1.4) and (1.5). Then every solution $h \in \mathscr{W}^{1,2}(\Omega)$ with nonnegative Jacobian is locally Lipschitz continuous but not necessarily $\mathscr{C}^{1}$-smooth.

A special case of Theorem 1.2 deserves a separate mention because it covers the variant of (1.3) with not necessarily analytic right-hand side $\phi$. 
Theorem 1.3. Let $h \in \mathscr{W}^{1,2}(\Omega)$ be a mapping with nonnegative Jacobian. Suppose that the Hopf product $h_{z} \overline{h_{\bar{z}}}$ is bounded and Hölder continuous. Then $h$ is locally Lipschitz but not necessarily $\mathscr{C}^{1}$-smooth.

More specific statements, including gradient estimates near $\partial \Omega$, are presented in Theorem 3.1. Examples of variational problems leading to equations in Theorem 1.2 are provided in Section 2.

Our proofs draw upon the theory of Beltrami equations combined with methods of topology, a technique originated in the theory of general nonlinear first order elliptic systems $[3,5,10,15]$. Initially, for the linear elliptic system

$$
h_{\bar{z}}=\mu h_{z}+\nu \overline{h_{\bar{z}}}, \quad|\mu|+|\nu| \leqslant k<1
$$

the solutions are quasiregular, a concept firmly rooted in the geometry of analytic functions. While it might sound trivial, the observation that the difference of two solutions is again quasiregular is deep and useful, for it provides us with powerful topological tools to obtain existence, uniqueness and regularity of solutions. And this was exactly a blueprint for the definition of ellipticity of fully nonlinear Beltrami type equations,

$$
h_{\bar{z}}=\mathcal{H}\left(z, h_{z}\right), \quad\left|\mathcal{H}\left(z, \xi_{1}\right)-\mathcal{H}\left(z, \xi_{2}\right)\right| \leqslant k\left|\xi_{1}-\xi_{2}\right|, \quad 0 \leqslant k<1
$$

Because of nonlinearity the difference of two solutions need not solve the same equation but it does solve another elliptic equation and, as such, satisfies the distortion inequality

$$
\left|f_{\bar{z}}-g_{\bar{z}}\right| \leqslant k\left|f_{z}-g_{z}\right|
$$

meaning that $f-g$ is quasiregular, see Definition 3.2.

A chief distinction from the elliptic cases discussed above is that the solutions of (1.6) need not be quasiregular. However, we will construct a continuous family $\left\{F^{\lambda}\right\}_{\lambda \in \mathbb{C}}$ of "good" solutions of (1.6) such that $F^{\lambda}-$ $h$ are quasiregular. After that we appeal to the topological properties of quasiregular mappings.

We believe that these ideas will have applications to even more general PDEs than those in our paper. An interested reader is referred to recent papers by D. Faraco, B. Kirchheim and L. Székelyhidi [13, 21] which also combine the theory of quasiregular mappings with topological arguments.

\section{INNER-VARIATIONAL EQUATIONS}

Let us consider the energy integral for mappings $h: \Omega \rightarrow \mathbb{C}$

$$
\mathscr{E}[h]=\iint_{\Omega} \mathbf{E}\left(z, h, h_{z}, h_{\bar{z}}\right) \mathrm{d} x_{1} \mathrm{~d} x_{2}, \quad z=x_{1}+i x_{2}
$$

where $\mathbf{E}=\mathbf{E}(z, w, \xi, \zeta)$ is a given stored-energy function. From the point of view of Geometric Function Theory the mappings $h$ must take $\Omega$ onto a designated domain $\Omega^{*}$. In the elasticity theory these domains are referred to as the reference configuration and deformed configuration, respectively. In the neo-Hookean model of hyperelasticity the stored-energy function blows 
up when the Jacobian of $h$ approaches zero. Thus one is looking for mapping $h$ with positive Jacobian determinant which minimizes the energy. It is not always the case that the minimizers satisfy the Euler-Lagrange equation. However, it is legitimate to perform the inner variation of the energy integral.

Given any test function $\eta \in \mathscr{C}_{0}^{\infty}(\Omega)$ and a complex parameter $t$, small enough so that the map $z \mapsto z+t \eta(z)$ represents a diffeomorphism of $\Omega$ onto itself, consider the inner variation $h^{t}(z)=h(z+t \eta)$ and its energy

$$
\mathscr{E}\left[h^{t}\right]=\iint_{\Omega} \mathbf{E}\left(z, h^{t}, h_{z}^{t}, h_{\bar{z}}^{t}\right) \mathrm{d} x_{1} \mathrm{~d} x_{2}
$$

First we make a substitution $w=z+t \eta(z)$ and then differentiate to obtain an integral form of the equilibrium equation $\left.\frac{\partial}{\partial t}\right|_{t=0} \mathscr{E}\left[h^{t}\right]=0$. We eliminate $\eta$ through integration by parts to arrive at what is called the inner-variational equation

$$
\frac{\partial}{\partial \bar{z}}\left[h_{z} \mathbf{E}_{\zeta}+\overline{h_{\bar{z}}} \mathbf{E}_{\bar{\xi}}\right]+\frac{\partial}{\partial z}\left[h_{z} \mathbf{E}_{\xi}+\overline{h_{\bar{z}}} \mathbf{E}_{\bar{\zeta}}-\mathbf{E}\right]+\mathbf{E}_{z}=0
$$

Here the subscripts under $\mathbf{E}$ stand for complex partial derivatives of $\mathbf{E}=$ $\mathbf{E}(z, w, \xi, \zeta)$. The partial derivatives $\frac{\partial}{\partial z}$ and $\frac{\partial}{\partial \bar{z}}$ are understood in the sense of distributions. Note that $\mathbf{E}_{w}$ does not enter this equation; meaning that $\mathbf{E}$ is not required to be differentiable with respect to the $w$-variable. We say that $h$ is inner stationary for $\mathscr{E}$ if it satisfies the equation (2.2), regardless of whether $h$ is energy-extremal or not.

2.1. Dirichlet integral. The most basic example is the Dirichlet integrand $\mathbf{E}=|\xi|^{2}+|\zeta|^{2}$ and the associated Hopf-Laplace equation (1.3),

$$
\frac{\partial}{\partial \bar{z}}\left(h_{z} \overline{h_{\bar{z}}}\right)=0, \quad \text { for } h \in \mathscr{W}_{\mathrm{loc}}^{1,2}(\Omega)
$$

2.2. Poincaré disk. More general equations arise in the theory of harmonic mappings between Riemann surfaces [19, 20, 23, 28]. In particular, let the target be the Poincaré disk. This is the unit disk $\mathbb{D}=\{w \in \mathbb{C}:|w|<1\}$ equipped with the hyperbolic metric $\mathrm{d} s=\frac{|\mathrm{d} w|}{1-|w|^{2}}$. The associated Dirichlet integral

$$
\mathscr{E}[h]=\iint_{\Omega} \frac{\left|h_{z}\right|^{2}+\left|h_{\bar{z}}\right|^{2}}{\left(1-|h|^{2}\right)^{2}} \mathrm{~d} x_{1} \mathrm{~d} x_{2}
$$

is certainly infinite for homeomorphisms $h: \Omega \stackrel{\text { onto }}{\longrightarrow} \mathbb{D}$ in the Sobolev space $\mathscr{W}_{\text {loc }}^{1,2}(\Omega, \mathbb{D})$. Nonetheless, it is interesting to examine the inner-variational equation and all its solutions, not necessarily homeomorphisms.

$$
\frac{\partial}{\partial \bar{z}} \frac{h_{z} \overline{h_{\bar{z}}}}{\left(1-|h|^{2}\right)^{2}}=0, \quad \text { for } h \in \mathscr{W}_{\mathrm{loc}}^{1,2}(\Omega, \mathbb{D})
$$

For slightly greater generality we consider the weighted Dirichlet integral

$$
\mathscr{E}[h]=\iint_{\Omega}\left(\left|h_{z}\right|^{2}+\left|h_{\bar{z}}\right|^{2}\right) \rho(z, h) \mathrm{d} x_{1} \mathrm{~d} x_{2}
$$


and its inner-variational equation

$$
\frac{\partial}{\partial \bar{z}}\left[\rho(z, h) h_{z} \overline{h_{\bar{z}}}\right]=\rho_{z}(z, h)\left(\left|h_{z}\right|^{2}+\left|h_{\bar{z}}\right|^{2}\right), \quad \text { for } h \in \mathscr{W}_{\mathrm{loc}}^{1,2}(\Omega, \mathbb{D})
$$

Theorem 2.1. Suppose $\rho=\rho(z, w) \geqslant 1$ is Lipschitz continuous in the $z$-variable and Hölder continuous in the $w$-variable. If $h \in \mathscr{W}_{\mathrm{loc}}^{1,2}(\Omega, \mathbb{D})$ is a solution of (2.3) with nonnegative Jacobian, then $h$ is locally Lipschitz continuous.

Proof. Generally speaking we are dealing with a nonhomogeneous CauchyRiemann equation

$$
\frac{\partial U}{\partial \bar{z}}=u, \quad \text { where } \quad U=\rho(z, h) h_{z} \overline{h_{\bar{z}}}, \quad u=\left(\left|h_{z}\right|^{2}+\left|h_{\bar{z}}\right|^{2}\right) \rho_{z}(z, h)
$$

The equation is elliptic, so we gain some regularity properties of $U$ and $u$. At the beginning we only know that $U, u \in \mathscr{L}_{\text {loc }}^{1}(\Omega)$. We shall recurrently improve integrability properties of these terms. First observe that $U$, having $\frac{\partial}{\partial \bar{z}}$-derivative in $\mathscr{L}_{\text {loc }}^{1}(\Omega)$, lies in $\mathscr{L}_{\text {loc }}^{s}(\Omega)$ for every exponent $1<s<2$. Then, in view of pointwise inequality $\left|h_{\bar{z}}\right|^{2} \leqslant \rho(z, h)\left|h_{z}\right|\left|h_{\bar{z}}\right|=|U|$, we see that $\left|h_{\bar{z}}\right|^{2} \in \mathscr{L}_{\mathrm{loc}}^{s}(\Omega)$. This implies that also $\left|h_{z}\right|^{2} \in \mathscr{L}_{\mathrm{loc}}^{s}(\Omega)$. In this way we gain higher integrability of the right hand side of (2.4); namely, $u=\left(\left|h_{z}\right|^{2}+\left|h_{\bar{z}}\right|^{2}\right) \rho_{z}(z, h) \in \mathscr{L}_{\text {loc }}^{s}(\Omega)$, because $\rho_{z}(z, h)$ is bounded. Now equation (2.4) places $U$ in the space $\mathscr{L}_{\text {loc }}^{\frac{2 s}{2-s}}(\Omega)$. This, in view of $\left|h_{\bar{z}}\right|^{2} \leqslant|U|$, yields $\left|h_{\bar{z}}\right|^{2} \in \mathscr{L}_{\text {loc }}^{\frac{2 s}{2-s}}(\Omega)$. Hence $\left|h_{\bar{z}}\right|^{2} \in \mathscr{L}_{\text {loc }}^{\frac{2 s}{2-s}}(\Omega)$ as well. Thus we gained more integrability of $u$; namely, $u \in \mathscr{L}_{\text {loc }}^{p}(\Omega)$, with $p=\frac{2 s}{2-s}>2$. We again turn back to equation (2.4). This time the equation yields Hölder continuity of $U$; that is, $U \in \mathscr{C}_{\text {loc }}^{\alpha}(\Omega)$ with $\alpha=1-\frac{2}{p}>0$. Let us write the equation as

$$
h_{z} \overline{h_{\bar{z}}}=\frac{\psi(z)}{\rho(z, h)}, \quad \text { where } \quad \psi \in \mathscr{C}_{\mathrm{loc}}^{\alpha}(\Omega)
$$

We observe that $h$ is also locally Hölder continuous, because $h_{\bar{z}} \in \mathscr{L}_{\mathrm{loc}}^{2 p}(\Omega)$ with exponent $2 p>2$. The conclusion is that the Hopf product $h_{z} \overline{h_{\bar{z}}}$ is a Hölder continuous function. By Theorem 1.3, $h$ is locally Lipschitz.

2.3. An application to nonlinear elasticity. We now turn to some fairly general energy integrals of interest in nonlinear elasticity. Given two bounded domains $\Omega$ and $\Omega^{*}$ in $\mathbb{C}$, we consider mappings $h: \Omega \rightarrow \Omega^{*}$ of Sobolev class $\mathscr{W}^{1,2}(\Omega)$ whose Jacobian determinant $J_{h} \stackrel{\text { def }}{=} \operatorname{det} D h=\left|h_{z}\right|^{2}-\left|h_{\bar{z}}\right|^{2}$ is nonnegative. In nonlinear elasticity of isotropic materials one considers the energy of $h$ of the form

$$
\mathscr{E}[h]=\iint_{\Omega} W\left(z, h,\left|h_{z}\right|^{2},\left|h_{\bar{z}}\right|^{2}\right)
$$

Specifically, neo-Hookean models of elasticity [6] deal with the integrands $W$ which blow up as the Jacobian determinant approaches zero. For the sake of simplicity we forgo the dependence of $W$ on the $z$ and $h$ variables. 
The interested reader may generalize our considerations by including $z$ and $h$ variables to the integrand, like in $\S 2.2$. For the energy integrand

$$
\mathbf{E}(D h)=W\left(\left|h_{z}\right|^{2},\left|h_{\bar{z}}\right|^{2}\right)
$$

the inner-variational equation (2.2) simplifies as follows

$$
\frac{\partial}{\partial \bar{z}}\left[\left(W_{a}+W_{b}\right) h_{z} \overline{h_{\bar{z}}}\right]+\frac{\partial}{\partial z}\left[\left|h_{z}\right|^{2} W_{a}+\left|h_{\bar{z}}\right|^{2} W_{b}-W\right]=0
$$

where $W$ and its partial derivatives $W_{a}$ and $W_{b}$ are evaluated at $a=\left|h_{z}\right|^{2}$ and $b=\left|h_{\bar{z}}\right|^{2}$. To emphasize a possible neo-Hookean character of the integrand we express it as

$$
W(a, b)=\frac{F(a, b)}{(a-b)^{p-1}}
$$

So

$$
\mathscr{E}[h]=\iint_{\Omega} \mathbf{E}(D h)=\iint_{\Omega} \frac{F\left(\left|h_{z}\right|^{2},\left|h_{\bar{z}}\right|^{2}\right)}{\left(\left|h_{z}\right|^{2}-\left|h_{\bar{z}}\right|^{2}\right)^{p-1}}, \quad p \geqslant 1
$$

where $F=F(a, b)$ is defined and continuous in $\overline{\mathbb{O}}=\{(a, b): a \geqslant b \geqslant 0\}$ -the closure of the first octant $\mathbb{O} \stackrel{\text { def }}{=}\{(a, b): a>b>0\}$. Let us remove the corner of $\overline{\mathbb{O}}$ to introduce $\overline{\mathbb{O}}_{\circ} \stackrel{\text { def }}{=} \overline{\mathbb{O}} \backslash\{(0,0)\}$. As usual, we write

$$
|\nabla F|=\left|F_{a}\right|+\left|F_{b}\right| \text { and }\left|\nabla^{2} F\right|=\left|F_{a a}\right|+\left|F_{a b}\right|+\left|F_{b b}\right|
$$

We make the following standing assumptions on $F: \overline{\mathbb{O}} \rightarrow[0, \infty)$ :

$$
\begin{aligned}
& F \in \mathscr{C}(\overline{\mathbb{O}}) \cap \mathscr{C}^{2}(\overline{\mathbb{O}} \backslash\{(0,0)\}) \\
& F \text { is homogeneous of degree } p \text {; that is, } \\
& F(t a, t b)=t^{p} F(a, b) \quad \text { for } t \geqslant 0, \quad a \geqslant b \geqslant 0 .
\end{aligned}
$$

Furthermore, for $a>b>0$, we assume that

$$
\begin{aligned}
(a+b)^{p} & \lesssim F \lesssim(a+b)^{p} \\
(a+b)^{p-1} \lesssim F_{a}+F_{b} \leqslant|\nabla F| \lesssim(a+b)^{p-1} & \\
& \left|\nabla^{2} F\right| \lesssim(a+b)^{p-2}
\end{aligned}
$$

Here the notation $\lesssim$ refers to an inequality with the implied constant (positive) which stays independent of $(a, b) \in \overline{\mathbb{O}}$.

Theorem 2.2. Let $h \in \mathscr{W}_{\mathrm{loc}}^{1,1}(\Omega)$ be an inner-stationary mapping for the energy integral (2.6) with $\mathscr{E}[h]<\infty$, where $F$ satisfies the conditions (2.7)(2.11). Then $h$ is locally Lipschitz continuous. Furthermore $\mathbf{E}(D h)$ is locally bounded.

Proof. First observe that

$$
\iint_{\Omega}|D h|^{2} \lesssim \mathscr{E}[h]<\infty
$$


hence $h \in \mathscr{W}^{1,2}(\Omega)$. Regarding inner variation, we apply formula $(2.5)$ to $W(a, b)=\frac{F(a, b)}{(a-b)^{p-1}}$ to see that $a W_{a}+b W_{b}-W=0$, because of $p$ homogeneity of $F$. The equation (2.5) takes the form

$$
\frac{\partial}{\partial \bar{z}}\left[\frac{F_{a}+F_{b}}{(a-b)^{p-1}} h_{z} \overline{h_{\bar{z}}}\right]=0
$$

where we note that

$$
\begin{aligned}
\left|\frac{F_{a}+F_{b}}{(a-b)^{p-1}} h_{z} \overline{h_{\bar{z}}}\right| & \lesssim \frac{(a+b)^{p-1} \sqrt{a b}}{(a-b)^{p-1}} \lesssim \frac{(a+b)^{p}}{(a-b)^{p-1}} \lesssim \frac{F(a, b)}{(a-b)^{p-1}} \\
& \lesssim \mathbf{E}(D h) \in \mathscr{L}^{1}(\Omega)
\end{aligned}
$$

Thus, by Weyl's lemma, (2.13) yields that

$$
\frac{F_{a}+F_{b}}{(a-b)^{p-1}} h_{z} \overline{h_{\bar{z}}}=\phi \quad \text { is an analytic function, actually in } \mathscr{L}^{1}(\Omega) .
$$

We also note at this point that in view of condition (2.10)

$$
\left|h_{\bar{z}}\right|^{2} \leqslant\left|h_{z}\right|\left|h_{\bar{z}}\right| \lesssim\left|\frac{F_{a}+F_{b}}{(a+b)^{p-1}} h_{z} \overline{h_{\bar{z}}}\right| \leqslant\left|\frac{F_{a}+F_{b}}{(a-b)^{p-1}} h_{z} \overline{h_{\bar{z}}}\right|=|\phi|
$$

Hence $h_{\bar{z}} \in \mathscr{L}_{\mathrm{loc}}^{\infty}(\Omega)$.

We are going to solve (2.14) for $h_{\bar{z}}$ in terms of $\phi$ and $h_{z}$, at least when $\left|h_{z}\right|$ is sufficiently large. Choose and fix an arbitrary subdomain $\Omega^{\prime} \Subset \Omega$ and let

$$
N^{2} \stackrel{\text { def }}{=}\|\phi\|_{\mathscr{L} \infty\left(\Omega^{\prime}\right)}<\infty
$$

Since $F_{a}+F_{b}$ is homogeneous of degree $p-1$, equation (2.14) can be written as

$$
\frac{F_{a}\left(1, k^{2}\right)+F_{b}\left(1, k^{2}\right)}{\left(1-k^{2}\right)^{p-1}} \frac{\overline{h_{\bar{z}}}}{h_{z}}=\frac{\phi}{h_{z}^{2}}
$$

where $0 \leqslant k=\frac{\left|h_{\bar{z}}\right|}{\left|h_{z}\right|} \leqslant 1 / 2$, provided

$$
\left|h_{z}\right| \geqslant 2 N \text {. }
$$

Taking the absolute values of both sides in the equation (2.16), we obtain

$$
\frac{F_{a}\left(1, k^{2}\right)+F_{b}\left(1, k^{2}\right)}{\left(1-k^{2}\right)^{p-1}} k=\frac{|\phi|}{\left|h_{z}\right|^{2}} \stackrel{\text { def }}{=} s
$$

The left hand side represents a $\mathscr{C}^{1}$-smooth function $\Phi=\Phi(k), 0<k \leqslant 1 / 2$. We see that

$$
\Phi^{\prime}(0)=F_{a}(1,0)+F_{b}(1,0) \gtrsim 1
$$

by the condition (2.10). Therefore (2.18) admits unique solution for $k$ close to 0 , say

$$
k=s \Gamma(s),
$$


provided $0 \leqslant k \leqslant k_{\circ} \leqslant 1 / 2$, where $\Gamma=\Gamma(s)$ is a $\mathscr{C}^{1}$-function in a small interval $0 \leqslant s \leqslant s_{\circ}$. Now the equation (2.16) reads as,

$$
\overline{h_{\bar{z}}}=\frac{\phi}{h_{z}} \Gamma\left(\frac{|\phi|}{\left|h_{z}\right|^{2}}\right), \quad \text { whenever }\left|h_{z}\right| \geqslant \frac{N}{k_{\circ}}
$$

Thus we arrive at the equation of the form (1.6), where

$$
\mathcal{H}(z, \xi)=\frac{\overline{\phi(z)}}{\bar{\xi}} \Gamma\left(\frac{|\phi(z)|}{|\xi|^{2}}\right), \quad \text { for }|\xi| \geqslant R \stackrel{\text { def }}{=} \frac{N}{k_{\circ}},
$$

All the conditions on $\mathcal{H}$ in Theorem 1.2 are satisfied, so

$$
|\nabla h| \in \mathscr{L}_{\mathrm{loc}}^{\infty}\left(\Omega^{\prime}\right) \text {. }
$$

It remains to estimate the integrand. We have the identity

$$
\frac{\mathbf{E}(D f)}{|\phi|+\left|h_{z}\right|}=\frac{F\left(1, k^{2}\right)}{\left(1-k^{2}\right)^{p-1}+\left[F_{a}\left(1, k^{2}\right)+F_{b}\left(1, k^{2}\right)\right] k}
$$

regardless of whenever $\left|h_{z}\right| \geqslant R$ holds or not, where $k=\frac{\left|h_{\bar{z}}\right|}{\left|h_{z}\right|} \in[0,1]$. The right hand side is bounded. Therefore,

$$
\mathbf{E}(D h) \lesssim|\phi|+\left|h_{z}\right| \in \mathscr{L}_{\mathrm{loc}}^{\infty}(\Omega)
$$

2.3.1. An example. The class of energies in (2.6) covers the following particular integral,

$$
\mathscr{E}_{\Omega}[h]=\iint_{\Omega} \frac{|D h|^{2 p}}{J_{h}^{p-1}}=\iint_{\Omega} \frac{\left(\left|h_{z}\right|^{2}+\left|h_{\bar{z}}\right|^{2}\right)^{p}}{\left(\left|h_{z}\right|^{2}-\left|h_{\bar{z}}\right|^{2}\right)^{p-1}}, \quad p \geqslant 1
$$

subject to homeomorphisms $h: \Omega \stackrel{\text { onto }}{\longrightarrow} \Omega^{*}$ in the Sobolev space $\mathscr{W}^{1,2}(\Omega)$. This case gains additional interest in Geometric Function Theory because the transition to the energy of the inverse mapping $f=h^{-1}: \Omega^{*} \stackrel{\text { onto }}{\longrightarrow} \Omega$ results in the $\mathscr{L}^{p}$-norm of the distortion function

$$
\mathscr{E}_{\Omega^{*}}[f]=\iint_{\Omega^{*}} K_{f}^{p}, \quad K_{f}=\frac{|D f|^{2}}{J_{f}} \geqslant 1
$$

We see that conformal mappings, for which $K_{f} \equiv 1$, are the absolute minimizers. In general, $\mathscr{L}^{p}$-integrability of the distortion function only guarantees that $f \in \mathscr{W}^{1, \frac{2 p}{p+1}}\left(\Omega^{*}\right)$. Indeed,

$$
\begin{aligned}
\iint_{\Omega^{*}}|D f|^{\frac{2 p}{p+1}} & =\iint_{\Omega^{*}} K_{f}^{\frac{p}{p+1}} J_{f}^{\frac{p}{p+1}} \leqslant\left(\iint_{\Omega^{*}} K_{f}^{p}\right)^{\frac{1}{p+1}}\left(\iint_{\Omega^{*}} J_{f}\right)^{\frac{p}{p+1}} \\
& =\left\|K_{f}\right\|_{\mathscr{L}^{p}\left(\Omega^{*}\right)}^{\frac{p}{p+1}} \cdot|\Omega|^{\frac{p}{p+1}}<\infty
\end{aligned}
$$

We do not pursue this matter further; see $[2,4,18,24]$ for more on the minimization of $\left\|K_{f}\right\|_{\mathscr{L}^{p}}$. 
2.3.2. Nonisotropic energies. The methods presented in this paper are also pertinent to some nonisotropic energies (2.1). By way of illustration, here is an example of such energy integral

$$
\mathscr{E}[h]=\iint_{\Omega} \mathbf{E}(D h)=\iint_{\Omega} \frac{F\left(\left|h_{z}\right|^{2},\left|h_{\bar{z}}\right|^{2}, 2 \operatorname{Re} h_{z} h_{\bar{z}}\right)}{\left(\left|h_{z}\right|^{2}-\left|h_{\bar{z}}\right|^{2}\right)^{p-1}}, \quad p \geqslant 1
$$

where $F=F(a, b, c)$ is a function of three real variables $a \geqslant b \geqslant 0$ and $c \in \mathbb{R}$. We assume, as in $\S 2.3$, that $F$ is homogeneous of degree $p$; that is,

$$
F(t a, t b, t c)=t^{p} F(a, b, c)
$$

An elementary but tedious computation reveals that the inner-variational equation (2.2) takes the form :

$$
\frac{\left(F_{a}+F_{b}\right) h_{z} \overline{h_{\bar{z}}}+\left(h_{z}^{2}+{\overline{h_{\bar{z}}}}^{2}\right) F_{c}}{\left(\left|h_{z}\right|^{2}-\left|h_{\bar{z}}\right|\right)^{p-1}}=\phi \quad \text {-an analytic function in } \Omega .
$$

We leave it to the interested reader to complete this investigation by imposing precise, fairly minimal, conditions on $F=F(a, b, c)$ in order to implement Theorem 1.2. The conclusion is that $h$ is locally Lipschitz and the integrand $\mathbf{E}(D h)$ is locally bounded. An explicit example is:

$$
F(a, b, c)=(a+b)^{p}+\epsilon c^{2}(a+b)^{p-2}, \quad p \geqslant 2
$$

with $\epsilon>0$ sufficiently small.

\section{Elaborate statement And examples}

The nonnegativity of Jacobian for a solution of (1.6), under the structural conditions (1.4)-(1.5), implies

$$
\left\|h_{\bar{z}}\right\|_{\infty} \leqslant M+\sqrt{L}+R
$$

For the sake of greater generality, and clarity of the arguments as well, we reformulate our main result (Theorem 1.2) replacing the Jacobian condition with the boundedness of $h_{\bar{z}}$. Furthermore, we give a quantitative result with the sharp asymptotic bound on the gradient of $h$ near the boundary. Define

$$
\mathrm{OSC}_{\Omega}[h] \stackrel{\text { def }}{=} \sup _{a, b \in \Omega}|h(a)-h(b)|
$$

and

$$
|\nabla h(z)| \stackrel{\text { def }}{=}\left|h_{z}(z)\right|+\left|h_{\bar{z}}(z)\right|
$$

Theorem 3.1. To every equation (1.6) there corresponds a structural constant $\lambda_{\circ}=\lambda_{\circ}(\mathcal{H})$ such that the following holds. Suppose that a function $h \in \mathscr{W}^{1,2}(\Omega) \cap \mathscr{L}^{\infty}(\Omega)$ with $h_{\bar{z}} \in \mathscr{L}^{\infty}(\Omega)$ satisfies $(1.6)$. Then $h$ is locally Lipschitz. Moreover, for almost every $z \in \Omega$ we have

$$
|\nabla h(z)| \leqslant \frac{3}{r} \operatorname{OsC}_{\Omega}[h]+4\left\|h_{\bar{z}}\right\|_{\mathscr{L}^{\infty}(\Omega)}+6 \lambda_{\circ}
$$


where $r=\min \{\operatorname{dist}(z, \partial \Omega), 1\}$. In particular,

$$
\limsup _{z \rightarrow \partial \Omega}|\nabla h(z)| \cdot \operatorname{dist}(z, \partial \Omega) \leqslant 3 \operatorname{OSC}_{\Omega}[h]
$$

If, in addition, $h$ is continuous up to the boundary then

$$
\limsup _{z \rightarrow \partial \Omega}|\nabla h(z)| \cdot \operatorname{dist}(z, \partial \Omega)=0
$$

Prerequisites. Before proceeding to the proof of Theorem 3.1 we need some definitions.

Definition 3.2. A mapping $g \in W_{\text {loc }}^{1,2}(\Omega)$ is quasiregular if there exists a constant $k<1$ such that $\left|g_{\bar{z}}\right| \leqslant k\left|g_{z}\right|$ a.e. in $\Omega$. Such a mapping is called quasiconformal if it is also injective.

The Jacobian determinant $J_{g}=\left|g_{z}\right|^{2}-\left|g_{\bar{z}}\right|^{2}$ of a quasiregular mapping is clearly nonnegative. It is well-known that a nonconstant quasiregular mapping is both open and discrete, see [3, Corollary 5.5.2] or [22, Chapter $\mathrm{VI}$. In the sequel we shall appeal to the following topological fact which relates the cardinality of preimages with the topological degree [14, Theorem 4] and [26, Proposition I.4.10].

Lemma 3.3. Let $\Omega \subset \mathbb{C}$ be a bounded domain and $G: \bar{\Omega} \rightarrow \mathbb{C}$ an orientation preserving continuous mapping that is open and discrete in $\Omega$. Then for every $v \notin G(\partial \Omega)$ we have

$$
\operatorname{Card}\{z \in \Omega: G(z)=v\} \leqslant \operatorname{deg}_{\Omega}[v ; G]
$$

Examples. Here we provide examples that demonstrate the sharpness of both assumptions and conclusions of our theorems.

The first example shows that even the most basic of our equations,

$$
h_{z} \overline{h_{\bar{z}}}=-1
$$

admits $\mathscr{W}^{1,2}$-solutions with $J_{h} \geqslant 0$ that are not $\mathscr{C}^{1}$-smooth.

Example 3.4. Let $\Omega=\{z:|z-1|<1 / 2\}$. For $z \in \Omega \backslash[1,3 / 2)$ we define

$$
h(z)=\frac{3}{2}\left(\bar{z}^{2 / 3}-z^{2 / 3}\right)+\left(1-z^{2 / 3}\right)^{3 / 2}+\left(1-\bar{z}^{2 / 3}\right)^{3 / 2}
$$

using the principal branch of the power function. Then

$$
\begin{aligned}
& h_{z}=-z^{-1 / 3}-z^{-1 / 3}\left(1-z^{2 / 3}\right)^{1 / 2} \\
& h_{\bar{z}}=\bar{z}^{-1 / 3}-\bar{z}^{-1 / 3}\left(1-\bar{z}^{2 / 3}\right)^{1 / 2}
\end{aligned}
$$

Therefore, $h_{z} \overline{h_{\bar{z}}}=-1$. Note $h$ has a Lipschitz extension to $\Omega$, in fact $h=0$ on the interval $[1,3 / 2)$. One can also check that $J_{h} \geqslant 0$ a.e. However, $h \notin \mathscr{C}^{1}(\Omega)$, because $z^{1 / 3}\left(h_{z}+\overline{h_{\bar{z}}}\right)=2\left(1-z^{2 / 3}\right)^{1 / 2}$ fails to be continuous. 
A much simpler example can be given if one does not insist on the special Hopf-Laplace structure $h_{z} \overline{h_{\bar{z}}}$, arising from the Dirichlet energy integral. Let

$$
h(z)= \begin{cases}3 z, & \operatorname{Im} z \geqslant 0 \\ 2 z+\bar{z}, & \operatorname{Im} z<0\end{cases}
$$

This is a bi-Lipschitz mapping of $\mathbb{C}$ which is not $\mathscr{C}^{1}$-smooth but satisfies the equation

$$
h_{\bar{z}}=\frac{6}{h_{z}}-2,
$$

as well as any equation of the form $h_{\bar{z}}=\mathcal{H}\left(h_{z}\right)$ with $\mathcal{H}(2)=1$ and $\mathcal{H}(3)=0$.

Theorem 3.1 also encompasses other Hopf type products such as

$$
\begin{array}{ll}
h_{z} h_{\bar{z}}=\phi, & \phi \in \mathscr{C}^{\alpha}(\Omega) \\
\left|h_{z}\right| h_{\bar{z}}=\psi, & \psi \in \mathscr{C}^{\alpha}(\Omega),
\end{array}
$$

but fails for some Hopf type products, like in the pseudo Hopf-Laplace equation below.

Example 3.5. The equation

$$
h_{z}\left|h_{\bar{z}}\right|=1
$$

admits a solution that is quasiconformal but not locally Lipschitz.

Proof. We look for $h$ in the form

$$
h(z)=2 z \psi(-2 \log |z|), \quad|z| \leqslant 1
$$

where $\psi:[0, \infty) \rightarrow[1, \infty)$ is a strictly increasing function with $\psi(0)=1$. Since

$$
h_{z}=2 \psi(-2 \log |z|)-2 \psi^{\prime}(-2 \log |z|) \quad h_{\bar{z}}=-\frac{2 z}{\bar{z}} \psi^{\prime}(-2 \log |z|)
$$

the function $\psi$ must satisfy the differential equation

$$
\left(\psi(t)-\psi^{\prime}(t)\right) \psi^{\prime}(t)=\frac{1}{4}, \quad 0<t<\infty .
$$

Rewriting (3.7) as an equation for the inverse function

$$
\frac{d t}{d \psi}=\frac{1}{2}\left(\psi+\sqrt{\psi^{2}-1}\right), \quad t(1)=0,
$$

we arrive at

$$
t=\psi^{2}-1+\psi \sqrt{\psi^{2}-1}-\log \left(\psi+\sqrt{\psi^{2}-1}\right) .
$$

The equation (3.9) determines a differentiable strictly increasing function $\psi:[0, \infty) \rightarrow[1, \infty)$, since the right hand side of $(3.8)$ is positive. Note that $\psi(t) \rightarrow \infty$ as $t \rightarrow \infty$, hence $h$ is not Lipschitz in any neighborhood of the origin. In view of equation (3.7) this implies $\psi^{\prime}(t) \rightarrow 0$ as $t \rightarrow \infty$. It now follows from (3.6) that $h$ is quasiconformal in a neighborhood of the origin; in particular $J_{h}>0$ almost everywhere. 
The major difference between equations (3.3) and (3.4) is that the latter is not solvable for $h_{\bar{z}}$ in terms of $h_{z}$.

Our final example shows that the Hölder continuity of $z \mapsto \mathcal{H}(z, \xi)$ in Theorem 3.1 cannot be relaxed to continuity, even for the standard Hopf product.

Example 3.6. Let $h(z)=z \log \log |z|^{-2}$ for $|z|<1 / 2$. This mapping is an orientation preserving homeomorphism which belongs to $\mathscr{W}^{1, p}$ for all $p<\infty$. We compute

$$
h_{z}=\log \log \frac{1}{|z|^{2}}-\log ^{-1} \frac{1}{|z|^{2}} \quad \text { and } \quad h_{\bar{z}}=\frac{z}{\bar{z}} \log ^{-1} \frac{1}{|z|^{2}} .
$$

Clearly, $h_{z} \overline{h_{\bar{z}}}$ is continuous. However, $h$ is not Lipschitz.

Even for the most basic equation $h_{z} \overline{h_{\bar{z}}}=1$, allowing the Jacobian of $h$ to change sign destroys any hope for improved regularity [12].

\section{Model Case: the Hopf-Laplace equation}

In order to illustrate our ideas without getting into technicalities, we first take on stage the Hopf-Laplace equation

$$
h_{z} \overline{h_{\bar{z}}}=\phi(z), \quad \text { with } \phi \text { analytic in } \Omega \subset \mathbb{C}
$$

Theorem 4.1. Suppose that the Hopf product $h_{z} \overline{h_{\bar{z}}}=\phi(z) \quad$ is analytic and bounded in a domain $\Omega \subset \mathbb{C}$, for some $h \in \mathscr{W}^{1,2}(\Omega) \cap \mathscr{L}^{\infty}(\Omega)$ with $h_{\bar{z}} \in \mathscr{L}^{\infty}(\Omega)$. Then $h$ is locally Lipschitz. Moreover, for almost every $z \in \Omega$ we have

$$
|\nabla h(z)| \leqslant \frac{13 \mathrm{OSC}_{\Omega}[h]}{\operatorname{dist}(z, \partial \Omega)}+2\left\|h_{\bar{z}}\right\|_{\mathscr{L} \infty(\Omega)}+3\|\phi\|_{\mathscr{L} \infty(\Omega)}^{1 / 2}
$$

Proof. Finding good solutions to equation (4.1) presents no difficulty. First consider $\Omega=\mathbb{D}$-the unit disk, and assume that $\phi$ is bounded in $\mathbb{D}$. Denote by $\Phi=\Phi(z)$ the anti-derivative of $\phi$ such that $\Phi(0)=0$. Thus $\Phi_{\bar{z}}=0$ and $\Phi_{z}=\phi$. Clearly, $\Phi$ extends continuously to the closed unit $\operatorname{disk} \mathbf{D}=\overline{\mathbb{D}}$. The mappings $F^{\lambda}(z)=\lambda z+f^{\lambda}(z)$, where $f^{\lambda}(z)=\overline{\lambda^{-1} \Phi(z)}$ with complex parameter $\lambda \neq 0$, solve the same equation (4.1). Also note that $\left\|f^{\lambda}\right\|_{\infty} \leqslant|\lambda|^{-1}\|\phi\|_{\infty}$. A short computation reveals that the difference $g=g^{\lambda}(z)=F^{\lambda}(z)-h(z)$ is a $\mathscr{W}^{1,2}(\mathbb{D})$-solution to a linear Beltrami type equation

$$
g_{\bar{z}}(z)=\nu(z) \overline{g_{z}(z)}, \quad \nu(z)=\frac{-h_{\bar{z}}(z)}{\bar{\lambda}}, \quad|\nu(z)| \leqslant \frac{1}{2}
$$

whenever $|\lambda| \geqslant 2\left\|h_{\bar{z}}\right\|_{\infty}$. Now consider a continuous family of mappings $G^{\lambda}(z)=\frac{1}{\lambda} g^{\lambda}(z)=z+\frac{1}{\lambda}\left[f^{\lambda}(z)-h(z)\right]$. We have

$$
\left|G^{\lambda}(z)-z\right| \leqslant \frac{\|\phi\|_{\infty}}{|\lambda|^{2}}+\frac{\|h\|_{\infty}}{|\lambda|}<\frac{1}{3}
$$


provided $|\lambda| \geqslant 2 \sqrt{\|\phi\|_{\infty}}$ and $|\lambda| \geqslant 13\|h\|_{\infty}$. This shows, in particular, that $G^{\lambda}$ is a nonconstant quasiregular mapping, thus orientation-preserving, open and discrete. At this point we appeal to a Rouché type lemma.

Lemma 4.2. Let $G=G^{\lambda}(z)$ be a continuous family of mappings $G^{\lambda}$ : $\mathbf{D} \rightarrow \mathbb{C}$ parametrized by complex numbers $\lambda$ with $\varrho \leqslant|\lambda| \leqslant \infty$, such that

(i) $\quad G^{\infty}(z) \equiv z$

(ii) $\left|G^{\lambda}(z)-z\right|<\frac{1}{3}$, for $z \in \mathbf{D}$ and $|\lambda| \geqslant \varrho$

(iii) For every $|\lambda| \geqslant \varrho$ the map $G^{\lambda}: \mathbb{D} \rightarrow \mathbb{C}$ is orientation preserving open and discrete.

Then, given any $z_{\circ} \in \frac{1}{3} \mathbf{D}$ and parameter $|\lambda| \geqslant \varrho$, the equation

$$
G^{\lambda}(z)=G^{\lambda}\left(z_{\circ}\right), \quad \text { for } z \in \mathbf{D} \text {; }
$$

admits exactly one solution $z=z_{\circ}$.

Proof of Lemma 4.2. For $|z|=1$, we see from (ii) that $\left|G^{\lambda}(z)\right|>\frac{2}{3}$. Fix any point $v$ of modulus $|v|<\frac{2}{3}$, so $v \notin G^{\lambda}(\partial \mathbf{D})$. Therefore, we have well defined topological degree of $v$, denoted by

$$
\operatorname{deg}_{\mathbb{D}}\left[v ; G^{\lambda}\right] \quad \text { for }|\lambda| \geqslant \varrho
$$

This is an integer-valued function, continuous in $\lambda$, thus constant. For $\lambda=\infty$ the degree is 1 , because $G^{\infty}(z) \equiv z$. Therefore

$$
\operatorname{deg}_{\mathbb{D}}\left[v ; G^{\lambda}\right]=1, \text { for all parameters }|\lambda| \geqslant \varrho .
$$

Now comes another topological fact concerning orientation preserving open discrete maps. It asserts that the cardinality of the preimage of $v$ does not exceed the degree, see Lemma 3.3. In symbols,

$$
0 \leqslant \operatorname{Card}\left\{z \in \mathbb{D}: G^{\lambda}(z)=v\right\} \leqslant \operatorname{deg}_{\mathbb{D}}\left[v ; G^{\lambda}\right]=1, \text { for all }|\lambda| \geqslant \varrho .
$$

This applies to the point $v=G^{\lambda}\left(z_{\circ}\right)$, because $\left|G^{\lambda}\left(z_{\circ}\right)\right| \leqslant\left|G^{\lambda}\left(z_{0}\right)-z_{\circ}\right|+$ $\left|z_{\circ}\right|<\frac{1}{3}+\frac{1}{3}=\frac{2}{3}$, by condition (ii). The lemma is established.

Returning to the proof of Theorem 4.1, we infer that the mappings $G^{\lambda}(z)=$ $\frac{1}{\lambda} g^{\lambda}(z)$ are injective in the disk $\frac{1}{3} \mathbf{D}$. So are the mappings $g^{\lambda}(z)=\lambda z+$ $\overline{\lambda^{-1}} \Phi(z)-h(z)$. This reads as follows:

$$
h\left(z_{1}\right)-h\left(z_{2}\right) \neq \lambda \cdot\left\{z_{1}-z_{2}+\frac{1}{|\lambda|^{2}}\left[\Phi\left(z_{1}\right)-\Phi\left(z_{2}\right)\right]\right\}
$$

for $z_{1} \neq z_{2}$ in the disk $\frac{1}{3} \mathbf{D}$. Letting $\lambda$ run over a circle of radius $|\lambda|$ we conclude that

$$
\left|h\left(z_{1}\right)-h\left(z_{2}\right)\right| \neq|\lambda| \cdot\left|z_{1}-z_{2}+\frac{1}{|\lambda|^{2}}\left[\Phi\left(z_{1}\right)-\Phi\left(z_{2}\right)\right]\right|
$$

This is possible only when

$$
\left|h\left(z_{1}\right)-h\left(z_{2}\right)\right|<|\lambda| \cdot\left|z_{1}-z_{2}+\frac{1}{|\lambda|^{2}}\left[\Phi\left(z_{1}\right)-\Phi\left(z_{2}\right)\right]\right|
$$


because the right hand side is continuous in $\lambda$ and the inequality (4.6) holds for large values of $|\lambda|$.

A conclusion is immediate; the solution $h$ is Lipschitz continuous in the disk $\frac{1}{3}$ D. Moreover,

$$
\|\nabla h\|_{\mathscr{L} \infty\left(\frac{1}{3} \mathbf{D}\right)} \leqslant|\lambda|+\frac{1}{|\lambda|}\|\phi\|_{\mathscr{L} \infty(\mathbf{D})}
$$

All the conditions we have encountered for the parameter $\lambda$ are satisfied if we set

$$
|\lambda|=\max \left\{\begin{array}{l}
2\left\|h_{\bar{z}}\right\|_{\mathscr{L} \infty(\mathbf{D})} \\
2\|\phi\|_{\mathscr{L}^{\infty}(\mathbf{D})}^{1 / 2} \\
13\|h\|_{\mathscr{L} \infty(\mathbf{D})}
\end{array}\right.
$$

Therefore,

$$
\|\nabla h\|_{\mathscr{L}^{\infty}\left(\frac{1}{3} \mathbf{D}\right)} \leqslant 2\left\|h_{\bar{z}}\right\|_{\mathscr{L}^{\infty}(\mathbf{D})}+13\|h\|_{\mathscr{L}^{\infty}(\mathbf{D})}+3\|\phi\|_{\mathscr{L}^{\infty}(\mathbf{D})}^{1 / 2}
$$

completing the analysis of the case $\Omega=\mathbb{D}$.

Now let $\Omega$ be a general domain. Suppose $\mathbf{B}(a, r)=\{z:|z-a| \leqslant r\}$ is a closed disk contained in $\Omega$ and $h$ is a solution to the Hopf-Laplace equation in $\Omega$. We scale down the variables to introduce a function $\hbar(z)=\frac{1}{r} h(r z+a)$ which satisfies the Hopf-Laplace equation $\hbar_{z} \overline{\hbar_{\bar{z}}}=\phi(r z+a)$ in the unit disk. Inequality (4.9), applied to $\hbar$, yields

$$
\|\nabla h\|_{\mathscr{L}^{\infty}\left(\frac{1}{3} \mathbf{B}\right)} \leqslant 2\left\|h_{\bar{z}}\right\|_{\mathscr{L}^{\infty}(\mathbf{B})}+\frac{13}{r}\|h\|_{\mathscr{L}^{\infty}(\mathbf{B})}+3\|\phi\|_{\mathscr{L}^{\infty}(\mathbf{B})}^{1 / 2}
$$

We are allowed to subtract any constant from $h$, given that $h$ appears in (4.1) only with its derivatives. The estimate (4.2) follows.

Corollary 4.3. Under the hypotheses of Theorem 4.1 we have

$$
\limsup _{z \rightarrow \partial \Omega}|\nabla h(z)| \cdot \operatorname{dist}(z, \partial \Omega) \leqslant 13 \mathrm{OSC}_{\Omega}[h]
$$

If, in addition, $h$ is continuous up to the boundary then

$$
\limsup _{z \rightarrow \partial \Omega}|\nabla h(z)| \cdot \operatorname{dist}(z, \partial \Omega)=0
$$

The property (4.11) may fail for solutions that are not continuous up to the boundary.

Example 4.4. Here is a bounded solution $h(z)=\bar{z}^{2}+\sin \log z$ in a halfdisk $\Omega=\{z: \operatorname{Re} z>0,|z|<1\}$ to the Hopf-Laplace equation

$$
h_{z} \overline{h_{\bar{z}}}=2 \cos \log z \quad \text {-analytic and bounded }
$$

This solution exhibits large oscillations arbitrarily close to the point $a=0 \in$ $\partial \Omega$. It is for this reason that $h$ fails to have a $\operatorname{limit} \lim _{z \rightarrow 0}(\operatorname{Re} z)|\nabla h(z)|$. 


\section{Outline of the Proof of Theorem 3.1}

The arguments presented for the proof of Theorem 4.1 contain many of the key ideas of the proof of our most general result, Theorem 3.1. The term $3\|\phi\|_{\mathscr{L}^{\infty}(\Omega)}^{1 / 2}$ in $(4.2)$ will be replaced by a number $\lambda_{\circ}=\lambda_{\circ}(\mathcal{H})$ which depends on the equation; that is, the conditions on $\mathcal{H}$. Let us emphasize that $\lambda_{\circ}$ will not depend on the solution $h$.

However, the situation is more intricate because we need to find a counterpart of the antiderivative of $\phi$. The proof of Theorem 4.1 suggests that we should look for the family $F^{\lambda}(z)=\lambda z+f^{\lambda}(z)$ that complies with the equation (1.6); that is, $f_{\bar{z}}=\mathcal{H}\left(z, \lambda+f_{z}\right)$. We must show that the latter equation admits a continuous family $\left\{f^{\lambda}\right\}$ of "good" solutions. The key is that $f^{\lambda}$ will enjoy uniform Lipschitz bounds, independent of $\lambda$. Then the proof of the Lipschitz regularity of $h$ will be carried out by topological arguments in much the same way as in the case of Hopf-Laplace equation.

We first consider the case $\Omega=\mathbb{D}$, and treat general domains $\Omega$ by rescaling. The sharpness of Lipschitz regularity was already demonstrated in Section 3.

\section{Good solutions}

We are looking for a family $\left\{f^{\lambda}: \lambda \in \mathbb{C},|\lambda| \geqslant \lambda_{\circ}\right\}$ of solutions to the equation

$$
f_{\bar{z}}=\mathcal{H}\left(z, \lambda+f_{z}\right)
$$

in the closed unit disk $\mathbf{D}=\{z:|z| \leqslant 1\}$, where $\mathcal{H}=\mathcal{H}(z, \xi)$ is continuous in $\mathbf{D} \times \widehat{\mathbb{C}}_{R}$ and satisfies the structural conditions (1.4) and (1.5) in $\Omega=\mathbb{D}$.

Proposition 6.1. There is $\lambda_{\circ}=\lambda_{\circ}(\alpha, L, M, R)$ and a family $\left\{f^{\lambda}\right\}_{|\lambda| \geqslant \lambda_{\circ}}$ of solutions to the equation (6.1) in $\mathbf{D}$ such that

$$
\begin{gathered}
f^{\lambda}(0)=0 \\
\left|f^{\lambda}\left(z_{1}\right)-f^{\lambda}\left(z_{2}\right)\right| \leqslant \lambda_{\circ} \cdot\left|z_{1}-z_{2}\right| \\
\left|f^{\lambda_{1}}(z)-f^{\lambda_{2}}(z)\right| \leqslant \lambda_{\circ} \cdot\left|\frac{\lambda_{1}-\lambda_{2}}{\lambda_{1} \cdot \lambda_{2}}\right|
\end{gathered}
$$

Furthermore, for every $z \in \mathbf{D}$ and $|\lambda| \geqslant \lambda_{\circ}$, we have

$$
\begin{aligned}
\left|\nabla f^{\lambda}(z)\right| & \leqslant \lambda_{\circ} \\
\left|f_{z}^{\lambda}(z)\right|,\left|f_{\bar{z}}^{\lambda}(z)\right| & \leqslant \frac{1}{2}\left|\lambda_{\circ}\right| \leqslant \frac{1}{2}|\lambda| \leqslant\left|\lambda+f_{z}^{\lambda}(z)\right|
\end{aligned}
$$

Proof. The proof of Proposition 6.1 is proceeded by an extension of equation (6.1) to the entire plane $\mathbb{C}$. 
6.1. Extension to $\mathbb{C}$. We set for all $z \in \mathbb{C}$ and $|\xi|>R$,

$$
\mathbf{H}(z, \xi)= \begin{cases}\mathcal{H}(z, \xi) & \text { if }|z| \leqslant 1 \\ (2-|z|) \cdot \mathcal{H}(1 / \bar{z}, \xi) & \text { if } 1 \leqslant|z| \leqslant 2 \\ 0 & \text { for }|z| \geqslant 2\end{cases}
$$

It is not difficult to see that inequalities (1.4) and (1.6) transmit to $\mathbf{H}$ as follows

$$
\begin{gathered}
\left|\mathbf{H}\left(z, \xi_{1}\right)-\mathbf{H}\left(z, \xi_{2}\right)\right| \leqslant L \cdot\left|\frac{1}{\xi_{1}}-\frac{1}{\xi_{2}}\right|, \text { for } z \in \mathbb{C} \text { and }\left|\xi_{1}\right|,\left|\xi_{2}\right|>R \\
\sup _{z \in \Omega}|\mathbf{H}(z, \xi)|+\sup _{z_{1} \neq z_{2}} \frac{\left|\mathbf{H}\left(z_{1}, \xi\right)-\mathbf{H}\left(z_{2}, \xi\right)\right|}{\left|z_{1}-z_{2}\right|^{\alpha}} \leqslant 6 M, \text { for }|\xi|>R
\end{gathered}
$$

The verification of (6.9) is a routine matter of the triangle inequality.

The desired solutions $f^{\lambda}: \mathbf{D} \rightarrow \mathbb{C}$ of (6.1) will be obtained as restrictions to the unit disk of the solutions, still denoted by $f=f^{\lambda}: \mathbb{C} \rightarrow \mathbb{C}$, of the extended equation

$$
f_{\bar{z}}=\mathbf{H}\left(z, \lambda+f_{z}\right)
$$

The advantage of passing to the extended equation lies in the use of singular integrals in the entire plane. We represent the solution in the form of the Cauchy transform of $\omega=f_{\bar{z}}$

$$
f(z)=\mathcal{C} \omega(z) \stackrel{\text { def }}{=} \frac{1}{\pi} \iint_{\mathbb{C}}\left[\frac{1}{z-\tau}+\frac{1}{\tau}\right] \omega(\tau)
$$

We search for the density function $\omega$ in a Besov space $\mathscr{B}_{\alpha}^{p}(\mathbb{C}) \subset \mathscr{L}^{p}(\mathbb{C})$, $p>\frac{2}{\alpha}>2$. The density function will be supported in the double disk $2 \mathbf{D}$. Recall the well known inequality [3, Theorem 4.3.13]

$$
|\mathcal{C} \eta(z)| \leqslant C_{p}|z|^{1-\frac{2}{p}}\|\eta\|_{p}, \quad z \in \mathbb{C}, \quad \eta \in \mathscr{L}^{p}(\mathbb{C})
$$

Thus,

$$
|f(z)| \leqslant C_{p}\|\omega\|_{p}, \quad \text { for } z \in \mathbf{D}
$$

6.2. The Besov Space $\mathscr{B}_{\alpha}^{p}(\mathbb{C})$. Let $0<\alpha<1$ be the Hölder exponent in (1.5). Let us choose and fix for the rest of our paper the integrability exponent

$$
p=\frac{3}{\alpha}>3
$$

The Besov space $\mathscr{B}_{\alpha}^{p}(\mathbb{C})$ consists of functions $\omega \in \mathscr{L}^{p}(\mathbb{C})$ which satisfy

$$
\|\omega\|_{\alpha, p} \stackrel{\text { def }}{=}\|\omega\|_{p}+\sup _{\tau \neq 0} \frac{\|\omega(\cdot+\tau)-\omega(\cdot)\|_{p}}{|\tau|^{\alpha}}<\infty
$$

We have a continuous imbedding $\mathscr{B}_{\alpha}^{p}(\mathbb{C}) \subset \mathscr{L}^{\infty}(\mathbb{C})$ together with a uniform bound [1, Theorem $7.34(\mathrm{c})]$ or [31, p. 131].

$$
\left\|f_{\bar{z}}\right\|_{\infty}=\|\omega\|_{\infty} \leqslant B_{p}\|\omega\|_{\alpha, p}
$$


6.3. Beurling-Ahlfors Transform. Complex derivative $f_{z}$ of the function in (6.11) is expressed by a singular integral, known as the BeurlingAhlfors transform

$$
f_{z}(z)=\mathcal{S} \omega(z) \stackrel{\text { def }}{=}-\frac{1}{\pi} \iint_{\mathbb{C}} \frac{\omega(\tau)}{(z-\tau)^{2}} \quad \text { where } \omega=f_{\bar{z}}
$$

We denote the norm of the operator $\mathcal{S}: \mathscr{L}^{p}(\mathbb{C}) \rightarrow \mathscr{L}^{p}(\mathbb{C})$ by $\mathcal{S}_{p}>1$. Thus we have

$$
\|\mathcal{S} \omega\|_{p} \leqslant \mathcal{S}_{p}\|\omega\|_{p}, \quad \text { hence } \quad\|\mathcal{S} \omega\|_{\alpha, p} \leqslant \mathcal{S}_{p}\|\omega\|_{\alpha, p}
$$

This combined with (6.16) yields

$$
\left\|f_{z}\right\|_{\infty}=\|\mathcal{S} \omega\|_{\infty} \leqslant \mathcal{S}_{p} B_{p}\|\omega\|_{\alpha, p}
$$

6.4. The structural parameter $\lambda_{\circ}$. We are now ready to reveal the conditions on the complex parameter $\lambda$; namely,

$$
|\lambda| \geqslant \lambda_{\circ} \stackrel{\text { def }}{=} \max \begin{cases}\sqrt{16 \mathcal{S}_{p}} \geqslant 4 & \text { condition }\left(\Lambda_{1}\right) \\ \sqrt{81 \mathcal{S}_{p} L} \geqslant \sqrt{8 \mathcal{S}_{p} L} & \text { condition }\left(\Lambda_{2}\right) \\ 120 \mathcal{S}_{p} B_{p} M & \text { condition }\left(\Lambda_{3}\right) \\ 3 R & \text { condition }\left(\Lambda_{4}\right) \\ 32 C_{p} L \geqslant 32 L & \text { condition }\left(\Lambda_{5}\right)\end{cases}
$$

These are not the optimal numerical values, but they are chosen for the clarity in subsequent computations.

6.5. Solving the Extended Equation. The equation (6.10) is now equivalent to the integral equation

$$
\omega=\mathbf{H}(z, \lambda+\mathcal{S} \omega)
$$

for a density function $\omega=\omega^{\lambda}(z)$, which we shall find uniquely in the set

$$
\mathfrak{B}=\left\{\omega:\|\omega\|_{\alpha, p} \leqslant 60 \cdot M\right\} \subset \mathscr{B}_{\alpha}^{p}(\mathbb{C}) \subset \mathscr{L}^{p}(\mathbb{C})
$$

This is a closed subset of $\mathscr{L}^{p}(\mathbb{C})$. We shall view $\mathfrak{B}$ as a complete metric space with respect to $\mathscr{L}^{p}$-norm. First observe that for each $\omega \in \mathfrak{B}$ we have

$$
\|\mathcal{S} \omega\|_{\infty} \leqslant 60 \mathcal{S}_{p} B_{p} M \leqslant \frac{1}{2} \lambda_{\circ},
$$

by (6.19) and condition $\left(\Lambda_{3}\right)$. This combined with conditions $\left(\Lambda_{3}\right)$ and $\left(\Lambda_{4}\right)$ yields

$$
|\lambda+\mathcal{S} \omega(z)| \geqslant \frac{1}{2}|\lambda| \geqslant \frac{1}{2} \lambda_{\circ}>R
$$

In particular, the equation $(6.21)$ is well defined on $\mathfrak{B}$. We now introduce a nonlinear operator $\mathrm{T}: \mathfrak{B} \rightarrow \mathscr{L}^{p}(\mathbb{C})$ by the rule

$$
\mathrm{T} \omega=\mathbf{H}(z, \lambda+\mathcal{S} \omega)
$$


Clearly, supp T $\omega \subset 2$ D . Note, by condition (6.9), that

$$
\|\mathrm{T} \omega\|_{p}=\|\mathbf{H}(z, \lambda+\mathcal{S} \omega)\|_{p} \leqslant 6 M \cdot\left\|\chi_{2 \mathbf{D}}\right\|_{p}=6(4 \pi)^{\alpha / 3} M \leqslant 18 \cdot M
$$

Next we estimate the difference quotient for $\mathrm{T} \omega$. To this effect we consider two cases

Case 1: $|\tau| \leqslant 1$. For all $z \in \mathbb{C}$, we can write

$$
\begin{aligned}
& |(\mathrm{T} \omega)(z+\tau)-(\mathrm{T} \omega)(z)| \\
& \leqslant|\mathbf{H}(z+\tau, \lambda+\mathcal{S} \omega(z+\tau))-\mathbf{H}(z+\tau, \lambda+\mathcal{S} \omega(z))| \\
& +|\mathbf{H}(z+\tau, \lambda+\mathcal{S} \omega(z))-\mathbf{H}(z, \lambda+\mathcal{S} \omega(z))|
\end{aligned}
$$

The first summand will be estimated by using (6.8) and (6.24),

$$
|\mathbf{H}(z+\tau, \lambda+\mathcal{S} \omega(z+\tau))-\mathbf{H}(z+\tau, \lambda+\mathcal{S} \omega(z))| \leqslant \frac{4 L}{|\lambda|^{2}}|\mathcal{S} \omega(z+\tau)-\mathcal{S} \omega(z)|
$$

For the second summand in (6.27) we use Hölder's estimate in (6.9)

$$
|\mathbf{H}(z+\tau, \lambda+\mathcal{S} \omega(z))-\mathbf{H}(z, \lambda+\mathcal{S} \omega(z))| \leqslant 6 M|\tau|^{\alpha}
$$

We add both summands to obtain

$$
|(\mathrm{T} \omega)(z+\tau)-(\mathrm{T} \omega)(z)| \leqslant \frac{4 L}{|\lambda|^{2}}|\mathcal{S} \omega(z+\tau)-\mathcal{S} \omega(z)|+6 M|\tau|^{\alpha} \cdot \chi_{3 \mathbf{D}}(z)
$$

The introduction of the factor $\chi_{3 \mathbf{D}}(z)$ is legitimate beacuse the left hand side vanishes outside the disk $3 \mathbf{D}$. We now compare the $\mathscr{L}^{p}(\mathbb{C})$-norms (with respect to $z$-variable) of both sides.

$$
\begin{aligned}
\|(\mathrm{T} \omega)(\cdot+\tau) & -(\mathrm{T} \omega)(\cdot) \|_{p} \\
& \leqslant \frac{4 L \mathcal{S}_{p}}{|\lambda|^{2}}\|\omega(\cdot+\tau)-\omega(\cdot)\|_{p}+6 M|\tau|^{\alpha} \cdot(9 \pi)^{1 / p}
\end{aligned}
$$

Hence, by the condition $\left(\Lambda_{2}\right)$,

$$
\frac{\|(\mathrm{T} \omega)(\cdot+\tau)-(\mathrm{T} \omega)(\cdot)\|_{p}}{|\tau|^{\alpha}} \leqslant \frac{4 L \mathcal{S}_{p}}{|\lambda|^{2}} \cdot 60 M+33 M \leqslant 36 M
$$

which together with (6.26) yields

$$
\|\mathrm{T} \omega\|_{p}+\frac{\|(\mathrm{T} \omega)(\cdot+\tau)-(\mathrm{T} \omega)(\cdot)\|_{p}}{|\tau|^{\alpha}} \leqslant 54 M \leqslant 60 M
$$

Case 2 : $|\tau| \geqslant 1$. It suffices to use rough $\mathscr{L}^{p}$-bound

$$
\|\mathrm{T} \omega\|_{p}+\frac{\|(\mathrm{T} \omega)(\cdot+\tau)-(\mathrm{T} \omega)(\cdot)\|_{p}}{|\tau|^{\alpha}} \leqslant 3\|\mathrm{~T} \omega\|_{p} \leqslant 54 M \leqslant 60 M
$$

Now we see that in both cases 1 and 2 we have a desired estimate $\|\mathrm{T} \omega\|_{\alpha, p} \leqslant$ $60 \mathrm{M}$, meaning that

$$
\mathrm{T}: \mathfrak{B} \rightarrow \mathfrak{B}
$$

Next we show that $\mathrm{T}: \mathfrak{B} \rightarrow \mathfrak{B}$ is a contraction in the $\mathscr{L}^{p}$-norm. Let $\omega_{1}, \omega_{2} \in \mathfrak{B}$. By (6.8) and (6.24) we see that 


$$
\begin{aligned}
\left\|\mathrm{T} \omega_{1}-\mathrm{T} \omega_{2}\right\|_{p} & \leqslant L\left\|\frac{1}{\lambda+\mathcal{S} \omega_{1}}-\frac{1}{\lambda+\mathcal{S} \omega_{2}}\right\|_{p} \\
& \leqslant \frac{4 L}{|\lambda|^{2}}\left\|\mathcal{S}\left(\omega_{1}-\omega_{2}\right)\right\|_{p} \\
& \leqslant \frac{4 L \mathcal{S}_{p}}{|\lambda|^{2}}\left\|\omega_{1}-\omega_{2}\right\|_{p}
\end{aligned}
$$

Thus the contraction constant is at most $\frac{1}{2}$, because of the condition $\left(\Lambda_{2}\right)$.

By virtue of Banach contraction principle the equation (6.21) has exactly one solution $\omega=\omega^{\lambda} \in \mathfrak{B}$. We define $f^{\lambda}(z) \stackrel{\text { def }}{=} \mathcal{C} \omega^{\lambda}(z)$.

It remains to examine the properties of $f^{\lambda}$. Using (6.16), (6.19) and (6.22) we estimate its derivatives as follows.

$$
\left\|f_{\bar{z}}^{\lambda}\right\|_{\infty} \leqslant B_{p}\left\|\omega^{\lambda}\right\|_{\alpha, p} \leqslant 60 B_{p} M<\frac{1}{2} \lambda_{\circ} \quad \text { for all } z \in \mathbb{C}
$$

and

$$
\left\|f_{z}^{\lambda}\right\|_{\infty}=\left\|\mathcal{S} f_{\bar{z}}^{\lambda}\right\|_{\infty} \leqslant \mathcal{S}_{p} B_{p}\left\|\omega^{\lambda}\right\|_{\alpha, p} \leqslant 60 \mathcal{S}_{p} B_{p} M \leqslant \frac{1}{2} \lambda_{\circ}, \quad z \in \mathbb{C}
$$

Thus

$$
\left\|\nabla f^{\lambda}\right\|_{\infty} \leqslant\left\|f_{z}^{\lambda}\right\|_{\infty}+\left\|f_{\bar{z}}^{\lambda}\right\|_{\infty}<\lambda_{\circ}
$$

which implies

$$
\left|f^{\lambda}(z)\right|<\lambda_{\circ}, \quad z \in \mathbf{D}
$$

This proves (6.3) in Proposition 6.1. Concerning continuity with respect to the parameter $\lambda$, let $\left|\lambda_{1}\right|,\left|\lambda_{2}\right| \geqslant \lambda_{\circ}$.

$$
\begin{aligned}
\left\|\omega^{\lambda_{1}}-\omega^{\lambda_{2}}\right\|_{p} & \leqslant L\left\|\frac{1}{\lambda_{1}+\mathcal{S} \omega^{\lambda_{1}}}-\frac{1}{\lambda_{2}+\mathcal{S} \omega^{\lambda_{2}}}\right\|_{\mathscr{L}^{p}(2 \mathbf{D})} \\
& \leqslant \frac{4 L}{\left|\lambda_{1} \lambda_{2}\right|}\left\|\left(\lambda_{1}-\lambda_{2}\right)+\mathcal{S}\left(\omega^{\lambda_{1}}-\omega^{\lambda_{2}}\right)\right\|_{\mathscr{L}^{p}(2 \mathbf{D})} \\
& \leqslant \frac{4 L}{\left|\lambda_{1} \lambda_{2}\right|}\left\|\lambda_{1}-\lambda_{2}\right\|_{\mathscr{L}^{p}(2 \mathbf{D})}+\frac{4 L \mathcal{S}_{p}}{\left|\lambda_{\circ}\right|^{2}}\left\|\omega^{\lambda_{1}}-\omega^{\lambda_{2}}\right\|_{\mathscr{L}^{p}(\mathbb{C})} \\
& \leqslant \frac{16 L}{\left|\lambda_{1} \lambda_{2}\right|}\left|\lambda_{1}-\lambda_{2}\right|+\frac{1}{2}\left\|\omega^{\lambda_{1}}-\omega^{\lambda_{2}}\right\|_{p}
\end{aligned}
$$

Hence

$$
\left\|\omega^{\lambda_{1}}-\omega^{\lambda_{2}}\right\|_{p} \leqslant \frac{32 L}{\left|\lambda_{1} \lambda_{2}\right|}\left|\lambda_{1}-\lambda_{2}\right|
$$

Finally, we recall the estimate (6.12) for Cauchy transform, $|\mathcal{C} \eta(z)| \leqslant C_{p}|z|^{1-\frac{2}{p}}\|\eta\|_{p}$ for all $z \in \mathbb{C}$, which we apply to $\eta=\omega^{\lambda_{1}}-\omega^{\lambda_{2}}$. This yields

$$
\left|f^{\lambda_{1}}(z)-f^{\lambda_{2}}(z)\right| \leqslant \frac{32 C_{p} L}{\left|\lambda_{1} \lambda_{2}\right|}\left|\lambda_{1}-\lambda_{2}\right| \leqslant \lambda_{\circ}\left|\frac{\lambda_{1}-\lambda_{2}}{\lambda_{1} \lambda_{2}}\right|, \quad \text { for all } z \in \mathbf{D}
$$


by the condition $\left(\Lambda_{5}\right)$, establishing the inequality (6.4) in Proposition 6.1.

We remark that $f^{\lambda}$ is $\mathscr{C}^{1, \alpha}$-smooth.

\section{The DIFFERENCE OF TWO SOLUTIONS}

In this section we consider the difference of two solutions of (1.6), namely the given solution $h$ and the good one $F^{\lambda}(z)=\lambda z+f^{\lambda}(z) e$ constructed in the previous section. Precisely, let

$$
g^{\lambda}(z)=\lambda z+f^{\lambda}(z)-h(z)
$$

We shall estimate the distortion of $g^{\lambda}$ under the assumption that

$$
\left\|h_{\bar{z}}(z)\right\|_{\mathscr{L} \infty(\mathbf{D})} \stackrel{\text { def }}{=} N<\infty
$$

To this effect we must impose additional bound from below on the complex parameters $\lambda$, which will now depend on the solution $h$.

Proposition 7.1. Let $h$ be as in Theorem 1.2 and $f^{\lambda}$ as in Proposition 6.1. If

$$
|\lambda| \geqslant 4 N+4 \lambda_{\circ}+R
$$

then the difference function $g^{\lambda}$ in (7.1) satisfies

$$
\left|g_{\bar{z}}^{\lambda}(z)\right| \leqslant \frac{1}{2}\left|g_{z}^{\lambda}(z)\right|, \quad \text { almost everywhere in } \mathbb{D}
$$

Proof. Fix any point $z \in \mathbb{D}$ at which $h_{z}$ and $h_{\bar{z}}$ are defined. To simplify writing we omit the superscript $\lambda$, so from now on $f=f^{\lambda}$ and $g=g^{\lambda}$. Thus

$$
g_{\bar{z}}=f_{\bar{z}}-h_{\bar{z}} \quad \text { and } \quad g_{z}=\lambda+f_{z}-h_{z}
$$

We begin with a simple case

Case 1: $\left|h_{z}\right| \leqslant R$. The computation goes as follows:

$$
\begin{aligned}
2\left|g_{\bar{z}}\right| & \leqslant 2\left(\left|f_{\bar{z}}\right|+\left|h_{\bar{z}}\right|\right) \leqslant 2\left(\frac{1}{2} \lambda_{\circ}+N\right) \\
& \leqslant|\lambda|-\frac{1}{2} \lambda_{\circ}-R \leqslant|\lambda|-\left|f_{z}\right|-\left|h_{z}\right| \leqslant\left|g_{z}\right|
\end{aligned}
$$

Case 2: $\left|h_{z}\right|>R$, so we may apply the equation (1.6). First, we have the identity

$$
\left(\lambda+f_{z}\right) g_{\bar{z}}=\left(g_{z}+h_{z}\right) g_{\bar{z}}=g_{z} g_{\bar{z}}+h_{z}\left(f_{\bar{z}}-h_{\bar{z}}\right)
$$

Hence, by (6.6)

$$
\frac{1}{2}|\lambda| \cdot\left|g_{\bar{z}}\right| \leqslant\left|\lambda+f_{z}\right| \cdot\left|g_{\bar{z}}\right| \leqslant\left|g_{z}\right| \cdot\left|g_{\bar{z}}\right|+\left|h_{z}\right| \cdot\left|f_{\bar{z}}-h_{\bar{z}}\right|
$$

Here in the right hand side we estimate the term $\left|g_{\bar{z}}\right|$ by using (7.2) and (6.5)

$$
\left|g_{\bar{z}}\right| \leqslant\left|h_{\bar{z}}\right|+\left|f_{\bar{z}}\right| \leqslant N+\frac{1}{2} \lambda_{\circ}
$$


For the second term in the right hand side of (7.5) we appeal to the equation (1.6) and the Lipschitz condition (1.4),

$$
\begin{aligned}
\left|h_{z}\right| \cdot\left|f_{\bar{z}}-h_{\bar{z}}\right| & =\left|h_{z}\right| \cdot\left|\mathcal{H}\left(z, \lambda+f_{z}\right)-\mathcal{H}\left(z, h_{z}\right)\right| \\
& \leqslant \frac{L\left|\lambda+f_{z}-h_{z}\right|}{\left|\lambda+f_{z}\right|}=\frac{L\left|g_{z}\right|}{\left|\lambda+f_{z}\right|} \leqslant \frac{2 L}{\lambda_{\circ}}\left|g_{z}\right| \leqslant \frac{1}{2} \lambda_{\circ}\left|g_{z}\right|
\end{aligned}
$$

For the last two estimates we have used (6.6) and condition $\left(\Lambda_{2}\right)$. Returning to (7.5), in view of $\left|\lambda+f_{z}\right| \geqslant \frac{1}{2}|\lambda|$, we arrive at the desired estimate

$$
|\lambda| \cdot\left|g_{\bar{z}}\right| \leqslant\left(2 N+2 \lambda_{\circ}\right) \cdot\left|g_{z}\right|
$$

That is,

$$
\left|g_{\bar{z}}\right| \leqslant \frac{2 N+2 \lambda_{\circ}}{|\lambda|} \cdot\left|g_{z}\right| \leqslant \frac{1}{2}\left|g_{z}\right|
$$

Corollary 7.2. Under the assumptions of Proposition 7.1 the mapping $g^{\lambda}$ is quasiregular, hence either constant or both open and discrete.

7.1. Topological degree. Consider a continuous family of mappings

$$
\left\{\begin{array}{l}
G^{\lambda}(z)=\frac{1}{\lambda} g^{\lambda}(z)=z+\frac{1}{\lambda}\left[f^{\lambda}(z)-h(z)\right], \text { for } z \in \mathbf{D} \text { and }|\lambda| \geqslant \sigma \\
G^{\infty}(z) \equiv z
\end{array}\right.
$$

where

$$
\sigma=3\|h\|_{\mathscr{L}^{\infty}(\mathbf{D})}+4\left\|h_{\bar{z}}\right\|_{\mathscr{L} \infty(\mathbf{D})}+5 \lambda_{\circ}
$$

From (6.31) we have the uniform bound

$$
\left|G^{\lambda}(z)-z\right|=\frac{1}{|\lambda|} \cdot\left|f^{\lambda}(z)-h(z)\right| \leqslant \frac{1}{|\lambda|} \cdot\left(\lambda_{\circ}+\|h\|_{\infty}\right)<\frac{1}{3}
$$

By Corollary 7.2 the mapping $G^{\lambda}: \mathbf{D} \rightarrow \mathbb{C}$ is quasiregular. Furthermore, it is nonconstant because for $\frac{1}{3}<|z|<1$ we have $\left|G^{\lambda}(z)\right|>|z|-\frac{1}{3}>$ $0=\left|G^{\lambda}(0)\right|$. Thus $G^{\lambda}(z)$ is open and discrete. By Lemma 4.2 we conclude that $G^{\lambda}\left(z_{1}\right) \neq G^{\lambda}\left(z_{2}\right)$, whenever $z_{1}$ and $z_{2}$ are distinct points in $\frac{1}{3} \mathbf{D}$ and $|\lambda| \geqslant \sigma$. This reads as follows

Corollary 7.3. For all complex parameters $\lambda$ with $|\lambda| \geqslant \sigma$ the mappings $g^{\lambda}(z)=\lambda z+f^{\lambda}(z)-h(z)$ are injective in the disk $\frac{1}{3} \mathbf{D}$; that is, for $z_{1} \neq z_{2}$ in $\frac{1}{3} \mathbf{D}$

$$
h\left(z_{1}\right)-h\left(z_{2}\right) \neq \lambda\left(z_{1}-z_{2}\right)+f^{\lambda}\left(z_{1}\right)-f^{\lambda}\left(z_{2}\right)
$$

We shall infer from this, using topological degree arguments, the following inequality

Lemma 7.4. For every circle $\mathbb{T}_{\rho}=\{\lambda:|\lambda|=\rho\}$ with $\rho \geqslant \sigma$ there exists $\lambda \in \mathbb{T}_{\rho}$ such that

$$
\left|h\left(z_{1}\right)-h\left(z_{2}\right)\right| \leqslant\left|\lambda\left(z_{1}-z_{2}\right)+f^{\lambda}\left(z_{1}\right)-f^{\lambda}\left(z_{2}\right)\right|
$$


Proof. This inequality certainly holds for large values of $\rho$. To simplify writing we denote $a=h\left(z_{1}\right)-h\left(z_{2}\right)$ and assume, as we may, that $a \neq 0$. We shall consider a family of mappings $\Phi_{\rho}^{a}: \mathbb{T} \rightarrow \mathbb{T}$, with parameter $\rho \geqslant \sigma$, given by

$$
\Phi_{\rho}^{a}\left(e^{i \theta}\right)=\frac{F\left(\rho e^{i \theta}\right)-a}{\left|F\left(\rho e^{i \theta}\right)-a\right|}, \text { where } F(\lambda)=\lambda \cdot\left(z_{1}-z_{2}\right)+f^{\lambda}\left(z_{1}\right)-f^{\lambda}\left(z_{2}\right)
$$

By virtue of the inequalities (7.8), each such mapping has well defined degree, denoted by $\operatorname{deg} \Phi_{\rho}^{a}$, also known as winding number. Letting the parameter $\rho$ vary we obtain an integer-valued continuous function in $\rho$, thus constant. We identify this constant by letting $\rho \rightarrow \infty$. The mappings converge uniformly to $\Phi_{\infty}^{a}: \mathbb{T} \rightarrow \mathbb{T}$, where $\Phi_{\infty}^{a}\left(e^{i \theta}\right) \stackrel{\text { def }}{=} \frac{z_{1}-z_{2}}{\left|z_{1}-z_{2}\right|} \cdot e^{i \theta}$. The degree of this limit map is equal to 1 . Hence we conclude that

$$
\operatorname{deg} \Phi_{\rho}^{a}=1, \text { for all parameters } \rho \geqslant \sigma
$$

We now fix $\rho \geqslant \sigma$ and move the point $a \neq 0$ to $\infty$ along the straight half-line $\{t a: t \geqslant 1\}$, to observe that for some $t \geqslant 1$ the point $t a$ lies in $F\left(\mathbb{T}_{\rho}\right)$. For if not, we would have well defined degree of the mappings $\Phi_{\rho}^{t a}: \mathbb{T} \rightarrow \mathbb{T}$, given by

$$
\Phi_{\rho}^{t a}\left(e^{i \theta}\right)=\frac{F\left(\rho e^{i \theta}\right)-t a}{\left|F\left(\rho e^{i \theta}\right)-t a\right|}
$$

By virtue of continuity with respect to the parameter $t$ we would have

$$
\operatorname{deg} \Phi_{\rho}^{t a}=\operatorname{deg} \Phi_{\rho}^{a}=1 \text {, for all } t \geqslant 1
$$

On the other hand letting $t \rightarrow \infty$ the mappings $\Phi_{\rho}^{t a}: \mathbb{T} \rightarrow \mathbb{T}$ converge uniformly to a constant map $\Phi_{\rho}^{\infty}=\frac{a}{|a|}$, whose degree is zero, in contradiction with the case $t=1$. Thus $t a \in F\left(\mathbb{T}_{\rho}\right)$, for some $t \geqslant 1$, meaning that

$$
t a=\lambda \cdot\left(z_{1}-z_{2}\right)+f^{\lambda}\left(z_{1}\right)-f^{\lambda}\left(z_{2}\right), \quad \text { for some } \lambda \in \mathbb{T}_{\rho}
$$

which yields the desired inequality (7.9).

\section{Proof of Theorem 3.1: The Final SteP}

We invoke Lemma 7.4 with $\rho=\sigma$, where $\sigma$ is given by (7.7). Thus

$\left|h\left(z_{1}\right)-h\left(z_{2}\right)\right| \leqslant t|a| \leqslant \sigma\left|z_{1}-z_{2}\right|+\left|f^{\rho e^{i \theta}}\left(z_{1}\right)-f^{\rho e^{i \theta}}\left(z_{2}\right)\right| \leqslant\left(\sigma+\lambda_{\circ}\right)\left|z_{1}-z_{2}\right|$

where the latter inequality follows from Proposition 6.1. In terms of the gradient of $h$ this reads as,

$$
|\nabla h(z)| \leqslant \sigma+\lambda_{\circ}=3\|h\|_{\mathscr{L}^{\infty}(\mathbf{D})}+4\left\|h_{\bar{z}}\right\|_{\mathscr{L}^{\infty}(\mathbf{D})}+6 \lambda_{\circ}
$$

The final procedure, like for the Hopf-Laplace equation, consists of rescaling the variables.

Let $\mathbf{B}(a, r)=\{z:|z-a| \leqslant r \leqslant 1\}$ be a closed disk contained in $\Omega$ and $h$ a solution to the equation $h_{\bar{z}}=\mathcal{H}\left(z, h_{z}\right)$ in $\Omega$. Consider the function $\hbar(z)=r^{-1} h(r z+a)$. It satisfies an equation $\hbar_{\bar{z}}=\widetilde{\mathcal{H}}\left(z, \hbar_{z}\right)$ in the unit 
disk, where $\widetilde{\mathcal{H}}(z, \xi)=\mathcal{H}(r z+a, \xi)$. We stress that $0<r \leqslant 1$, so all the conditions imposed on $\mathcal{H}(z, \xi)$, including $(6.9)$, transmit to $\tilde{\mathcal{H}}(z, \xi)$, with the same constants; easily verified. In other words the structural parameter $\lambda_{\circ}$ remains the same. Now inequality (8.1) applied to $\hbar$ yields

$$
\|\nabla h\|_{\mathscr{L}^{\infty}\left(\frac{1}{3} \mathbf{B}\right)} \leqslant \frac{3}{r}\|h\|_{\mathscr{L}^{\infty}(\mathbf{B})}+4\left\|h_{\bar{z}}\right\|_{\mathscr{L}^{\infty}(\mathbf{B})}+6 \lambda_{\circ}
$$

One may subtract a suitable constant from $h$, arriving at (3.2).

\section{REFERENCES}

1. R. A. Adams and J. J. F. Fournier, Sobolev spaces, Academic Press, Amsterdam, 2003.

2. K. Astala, T. Iwaniec, and G. Martin, Deformations of annuli with smallest mean distortion, Arch. Ration. Mech. Anal. 195 (2010), no. 3, 899-921.

3. K. Astala, T. Iwaniec, and G. Martin, Elliptic partial differential equations and quasiconformal mappings in the plane, Princeton University Press, Princeton, NJ, 2009.

4. K. Astala, T. Iwaniec, G. J. Martin, and J. Onninen, Extremal mappings of finite distortion, Proc. London Math. Soc. (3) 91 (2005), no. 3, 655-702.

5. K. Astala, T. Iwaniec, and E. Saksman, Beltrami operators in the plane, Duke Math. J. 107 (2001), no. 1, 27-56.

6. J. M. Ball, Convexity conditions and existence theorems in nonlinear elasticity, Arch. Rational Mech. Anal. 63 (1976/77), no. 4, 337-403.

7. J. M. Ball, Minimizers and the Euler-Lagrange equations, Trends and applications of pure mathematics to mechanics (Palaiseau, 1983), 1-4, Lecture Notes in Phys., 195, Springer, Berlin, 1984.

8. J. M. Ball, Some open problems in elasticity, Geometry, mechanics, and dynamics, 3-59, Springer, New York, 2002.

9. P. Bauman, N. C. Owen, and D. Phillips, Maximal smoothness of solutions to certain Euler-Lagrange equations from nonlinear elasticity, Proc. Roy. Soc. Edinburgh Sect. A 119 (1991), no. 3-4, 241-263.

10. B. Bojarski and T. Iwaniec, Analytical foundations of the theory of quasiconformal mappings in $R^{n}$, Ann. Acad. Sci. Fenn. Ser. A I Math. 8 (1983), no. 2, 257-324.

11. R. Courant, Dirichlet's principle, conformal mapping, and minimal surfaces, With an appendix by M. Schiffer. Springer-Verlag, New York-Heidelberg, 1950.

12. J. Cristina, T. Iwaniec, L. V. Kovalev, and J. Onninen, Lipschitz regularity for the Hopf-Laplace equation, arXiv:1011.5934.

13. D. Faraco and L. Székelyhidi, Tartar's conjecture and localization of the quasiconvex hull in $\mathbb{R}^{2 \times 2}$, Acta Math. 200 (2008), no. 2, 279-305.

14. E. Heinz, An elementary analytic theory of the degree of mapping in $n$-dimensional space, J. Math. Mech. 8 (1959) 231-247.

15. T. Iwaniec, Quasiconformal mapping problem for general nonlinear systems of partial differential equations, Symposia Mathematica, Vol. XVIII (Convegno sulle Transformazioni Quasiconformi e Questioni Connesse, INDAM, Rome, 1974), 501-517. Academic Press, London, 1976.

16. T. Iwaniec, N.-T. Koh, L. V. Kovalev, and J. Onninen, Existence of energy-minimal diffeomorphisms between doubly connected domains, Invent. Math., to appear.

17. T. Iwaniec, L. V. Kovalev, and J. Onninen, Hopf differentials and smoothing Sobolev homeomorphisms, Int. Math. Res. Not. IMRN, to appear.

18. M. Jordens and G. J. Martin, Deformations with smallest weighted $L^{p}$ average distortion and Nitsche type phenomena, J. Lond. Math. Soc., to appear.

19. J. Jost, A note on harmonic maps between surfaces, Ann. Inst. H. Poincaré Anal. Non Linéaire 2 (1985), no. 6, 397-405. 
20. J. Jost, Two-dimensional geometric variational problems, John Wiley \& Sons, Ltd., Chichester, 1991.

21. B. Kirchheim and L. Székelyhidi, On the gradient set of Lipschitz maps, J. Reine Angew. Math. 625 (2008), 215-229.

22. O. Lehto and K. I. Virtanen, Quasiconformal mappings in the plane, Springer-Verlag, New York-Heidelberg, 1973.

23. V. Marković, Harmonic diffeomorphisms of noncompact surfaces and Teichmüller spaces, J. London Math. Soc. (2) 65 (2002), no. 1, 103-114.

24. G. J. Martin, The Teichmüller problem for mean distortion, Ann. Acad. Sci. Fenn. Math. 34 (2009), no. 1, 233-247.

25. R. Moser, On a variational problem with non-differentiable constraints, Calc. Var. Partial Differential Equations 29 (2007), no. 1, 119-140.

26. S. Rickman, Quasiregular mappings, Springer-Verlag, Berlin, 1993.

27. E. Sandier and S. Serfaty, Limiting vorticities for the Ginzburg-Landau equations, Duke Math. J. 117 (2003), no. 3, 403-446.

28. R. Schoen, Analytic aspects of the harmonic map problem, in "Seminar on nonlinear partial differential equations" (Berkeley, Calif., 1983), 321-358, Math. Sci. Res. Inst. Publ., 2, Springer, New York, 1984.

29. J. Sivaloganathana and S. J. Spector, On irregular weak solutions of the energymomentum equations, Proc. R. Soc. Edinb. A 141 (2011), 193-204.

30. A. Taheri, Quasiconvexity and uniqueness of stationary points in the multidimensional calculus of variations, Proc. Amer. Math. Soc. 131 (2003), no. 10, 31013107.

31. H. Triebel, Theory of function spaces, Birkhäuser Verlag, Basel, 1983.

32. X. Yan, Maximal smoothness for solutions to equilibrium equations in $2 D$ nonlinear elasticity, Proc. Amer. Math. Soc. 135 (2007), no. 6, 1717-1724.

Department of Mathematics, Syracuse University, Syracuse, Ny 13244, USA and Department of Mathematics and Statistics, University of Helsinki, FinLAND

E-mail address: tiwaniec@syr.edu

Department of Mathematics, Syracuse University, Syracuse, NY 13244, USA

E-mail address: lvkovale@syr.edu

Department of Mathematics, Syracuse University, Syracuse, NY 13244, USA

E-mail address: jkonnine@syr.edu 